\title{
A better way to turn off neurons
}

Engineering of channelrhodopsin into a $\mathrm{Cl}^{-}$channel creates a powerful tool for light-mediated inhibition of neurons.

Use of light to control cellular activity has emerged as a powerful neuroscience research technique. Targeted neuronal expression of exogenous light-activated membrane proteins coupled with illumination, often called optogenetics, is used by the investigator to alter neuron electrical properties and signaling in a lightdependent manner. The $\mathrm{Na}^{+}$-conducting channelrhodopsin proteins are popular neuron activators, but investigation of neuronal circuits can be easier with inhibitors.

Now Peter Hegemann and colleagues in Germany have engineered channelrhodopsin into a $\mathrm{Cl}^{-}$channel they call chlorideconducting channelrhodopsin (ChloC), thus converting this popular neuronal activator into a powerful inhibitor. Although lightdependent $\mathrm{H}^{+}$pumps have been available to inhibit neurons for a number of years, their usage has been limited because they require continuous strong illumination to transport ions one at a time during each photon capture. In contrast, the ChloC channel allows many $\mathrm{Cl}^{-}$ions to flow down their concentration gradient during each photon capture. Furthermore, by introducing a mutation to slow the channel inactivation, the authors created a ChloC variant, slow ChloC, that achieved a similar electrophysiological effect with light intensities 10,000-fold lower than those required by ChloC.

Hegemann and colleagues tested the ability of ChloC and slow ChloC to inhibit neuronal spiking in CA1 pyramidal cells transfected by single-cell electroporation in organotypic hippocampal slice culture. Brief pulses of blue light inhibited 90\% and 100\% of evoked action potentials in ChloC- and slow ChloC-expressing neurons, respectively, as measured by electrophysiology.
The researchers also compared the performance of ChloC and slow ChloC to the light-driven proton pump, archaerhodopsin (Arch), which can currently be used to inhibit neurons. ChloC was slightly more inhibitory than Arch at low light intensities, but at the highest tested intensity, Arch surpassed it. Slow ChloC, however, needed only 10,000-fold lower light intensity to achieve this same level of inhibition, and at a light intensity midway between these extremes, no spiking could be induced in the slow ChloC-expressing neurons. This dramatic decrease in required irradiation for slow ChloC makes optogenetic inhibition of neuronal function a powerful alternative to activation.

\section{Daniel Evanko}

\section{RESEARCH PAPERS}

Wietek, J. et al. Conversion of channelrhodopsin into a light-gated chloride channel. Science 344, 409-412 (2014). 